\title{
Actitudes personales fundamentales para alcanzar el éxito empresarial en el Perú
}

\author{
Karen Weinberger Villarán \\ Candidata a Doctora en Economía y Dirección de Empresas por la \\ Universidad de Deusto, San Sebastián, España. \\ Profesora e Investigadora del Departamento Académico de Administración de la \\ Universidad del Pacífico, Lima, Perú.
}

\section{Resumen}

La mayoría de estudios sobre la actividad empresarial han tratado de explicar el papel que desempeña el empresario en la sociedad, así como las características psicológicas y no psicológicas que lo diferencian de los no empresarios. Si bien esta caracterización puede ser muy interesante para poder identificar potenciales empresarios, diversos estudios han demostrado que dichas características de los empresarios pueden ser sustancialmente diferentes o increíblemente similares entre ellos. Estas similitudes o diferencias pueden depender de aspectos económicos, sociales, culturales, políticos y legales, propios de un determinado ambiente empresarial; del tamaño de la empresa y del tipo de organización establecida; de las características personales y conocimientos de los empresarios; y de aspectos coyunturales o estructurales de una determinada industria. En consecuencia, es casi imposible hablar de un modelo de empresario o de un perfil universal del empresario.

A pesar de estas diferencias entre los mismos empresarios, toda empresa necesita de empresarios visionarios, capaces de identificar una oportunidad de negocio, de poner en marcha una iniciativa empresarial y de alcanzar el éxito empresarial satisfaciendo las necesidades de la sociedad. En ese sentido, el presente artículo da respuesta a las siguientes preguntas: ¿por qué algunos empresarios alcanzan el éxito y otros no? ¿Qué característica es fundamental para alcanzar el éxito empresarial? Para un grupo de empresarios peruanos exitosos, la formación y los conocimientos profesionales, así como la experiencia profesional, son necesarios e indispensables para llevar adelante una iniciativa empresarial. Sin embargo, la perseverancia es una característica sin la cual un empresario no alcanzaría el éxito en un país como el Perú.

\section{Palabras clave}

Empresario, Factores de éxito, Proceso emprendedor, Rol del empresario

\section{Introducción}

En la actualidad, no hay duda sobre la importancia de la creación de nuevas empresas y el crecimiento de las empresas ya existentes para el desarrollo económico y social de los países. Estas empresas no solo crean empleo, innovan productos, servicios y procesos en beneficio de actuales y potenciales clientes, sino que además contribuyen con el pago de impuestos en beneficio del Estado. En consecuencia, una actividad empresarial ética y responsable beneficia a todos los 
agentes involucrados: los accionistas obtienen mayores beneficios económicos, los empresarios tienen la oportunidad de ver cumplidos sus sueños, los empleados tienen más y mejores oportunidades de trabajo, los clientes ven sus necesidades plenamente satisfechas, los proveedores tienen una mayor demanda por sus productos y los competidores obtienen un aliciente para seguir mejorando e innovando día a día. Finalmente, el Estado, mediante una función redistributiva justa y equitativa de sus ingresos por recaudación de impuestos, contribuirá con el crecimiento y desarrollo de una sociedad más homogénea.

Si bien las empresas son importantes para el crecimiento y el desarrollo de un país, los empresarios, con actitud sensible e innovadora, desempeñan un papel preponderante en ella. Son los empresarios quienes ponen en marcha una nueva iniciativa empresarial, pero no todas logran sobrevivir y solo la minoría logra superar los tres años de vida. Muchos empresarios emprenden nuevos negocios, nuevas empresas, pero solo algunos logran desarrollar las empresas y hacerlas crecer. El Perú es un país con una alta tasa de nuevos emprendimientos, pero la mortandad de las empresas también es muy alta. Solo algunas logran convertirse en pequeñas, medianas y grandes empresas, constituyéndose en las verdaderas generadoras de riqueza económica para el país.

Dada la importancia de las empresas para el desarrollo socioeconómico de una nación, es importante responder a las siguientes preguntas: ¿por qué algunas empresas sí logran crecer de manera sostenida y otras no? ¿Qué es lo que las hace «diferentes»? ¿Qué es lo que caracteriza al empresario exitoso? Probablemente, el entorno general, sectorial o industrial podría afectar a algunas empresas más que a otras. Sin embargo, si comparamos a empresas de tamaños similares, que pertenecen a la misma industria o sector, la respuesta quizá no estará en el entorno ni en la industria; la respuesta estará en «el empresario».
Por lo tanto, si bien el entorno general o industrial puede afectar de manera positiva o negativa el desempeño de una empresa, es la capacidad del empresario la que marcará la diferencia entre el éxito y el fracaso. El empresario es la figura principal del proceso emprendedor.

\section{Objetivos del estudio}

Según un estudio realizado por Kantis et al., (2002) sobre entrepreneurship en economías emergentes, las características de los empresarios de América Latina y el Este de Asia son sustancialmente diferentes. Inclusive, dentro de cada uno de los continentes, las realidades entre los países también son distintas. En consecuencia, no es posible determinar un único perfil del empresario ni establecer con certeza cuáles son las características de los empresarios en el nivel global. Sin embargo, sí es posible conocer, en opinión de empresarios exitosos, ¿cuál es el factor primordial para el éxito empresarial en un determinado país y bajo qué circunstancias particulares podría alcanzarse?

Conscientes del rol del empresario y de la importancia de estudiar el proceso emprendedor para poder comprender el verdadero papel que desempeña el empresario, se decidió explorar si el empresario peruano se sentía exitoso, en qué medida la formación profesional adquirida y la experiencia profesional previa a la puesta en marcha del negocio contribuyeron con el éxito del empresario y, finalmente, cuáles son los factores $\mathrm{o}$ actitudes personales que, según los empresarios entrevistados, fueron esenciales para alcanzar el éxito empresarial.

\section{Marco conceptual}

\section{El rol del empresario ${ }^{1}$}

El rol del empresario en la sociedad ha sido descrito hace más de 250 años. Sin embargo, esta descripción ha ido cambiando en función de las

1. Se entiende por empresario al individuo que es capaz de descubrir una oportunidad de negocio y decide explotarla poniendo una empresa en marcha. Para ello, innova, arriesga su capital y asume la dirección de la misma. 
percepciones de los distintos investigadores sobre el tema, de los cambios que se presentaban en el entorno y de los resultados que se han obtenido en numerosos estudios. Probablemente, el modelo económico más representativo del entrepreneur fue el desarrollado por Joseph Schumpeter, en su obra The Theory of Economic Development, publicada en Alemania en 1911. Para el autor, el rol fundamental del empresario era innovar y crear. No obstante, han habido muchas otras interpretaciones de los diferentes roles del entrepreneur ${ }^{2}$, los cuales fueron resumidos por Hebert y Link (1988) como se muestra en el cuadro 1.
Como se puede apreciar, el empresario desempeñaba un rol económico como propietario-capitalista y generador de riqueza, una función creativa e innovadora al desarrollar nuevos y mejores productos, y un rol gerencial como coordinador de recursos en búsqueda de la eficiencia. Los investigadores pretendían tipificar a los empresarios en función del rol que desempeñaban en la sociedad y de sus características psicológicas y no psicológicas. En este sentido, la investigación se basó en conocer el rol del empresario en la sociedad, las características personales que lo diferenciaban de los no empresarios y las habilidades necesarias para cumplir con las funciones empresariales al interior de las empresas. Es de-

\section{Cuadro 1}

Interpretaciones del rol del entrepreneur

\begin{tabular}{|c|c|}
\hline Rol del entrepreneur & Autores \\
\hline $\begin{array}{l}\text { Es la persona que asume el riesgo asociado con la } \\
\text { incertidumbre }\end{array}$ & $\begin{array}{l}\text { Cantillon, Thünen, Mill, Hawley, Knight, Mises, Cole, } \\
\text { Shake }\end{array}$ \\
\hline Es la persona que provee el capital financiero & Smith, Turgot, Böhm Bawerk, Pigou, Mises \\
\hline Es un innovador & $\begin{array}{l}\text { Baudeau, Thünen, Schmoller, Sombart, Weber, } \\
\text { Schumpeter }\end{array}$ \\
\hline Es un tomador de decisiones & $\begin{array}{l}\text { Cantillon, Menger, Marschall, Wieser, Amasa Walker, } \\
\text { Francis Walker, Keynes, Miles, Shakle, Cole, Schultz }\end{array}$ \\
\hline Es un líder industrial & $\begin{array}{l}\text { Say, Sain-Simon, Amasa Walker, Francis Walker, } \\
\text { Marshall, Wieser, Sombart, Weber, Schumpeter }\end{array}$ \\
\hline Es un gerente o superintendente & Say, Mill, Marschall, Menger \\
\hline $\begin{array}{l}\text { Es un organizador y coordinador de los recursos } \\
\text { económicos }\end{array}$ & $\begin{array}{l}\text { Say, Walras, Wieser, Schmoller, Sombart, Weber, } \\
\text { Clark, Davenport, Schumpeter, Coase }\end{array}$ \\
\hline Es el propietario de una empresa & Quesnay, Wieser, Pigou, Hawley \\
\hline Es el que emplea los factores de producción & Amasa Walker, Francis Walker, Wieser, Keynes \\
\hline Es un individuo que contrata & Bentham \\
\hline Es un árbitro & Cantillon, Walras, Kirzner \\
\hline $\begin{array}{l}\text { Es un asignador de recursos entre distintas alter- } \\
\text { nativas de uso }\end{array}$ & Cantillon, Kirzner, Schultz \\
\hline
\end{tabular}

Fuente: elaboración propia.

2. Se entiende por emprendedor a aquella persona que es capaz de poner en marcha una innovación. 
cir, fue estudiado desde una perspectiva económica, psicológica y administrativa.

Durante los años 1970 y 1980 se produjeron cambios estructurales en la economía mundial como consecuencia de la crisis del petróleo, la recesión mundial; cambios tecnológicos; cambios en la orientación política de grandes potencias mundiales hacia ideologías orientadas al mercado; y el surgimiento de países con economías emergentes. Estos cambios crearon un ambiente de incertidumbre y desequilibrio, lo que generó un entorno positivo para la innovación empresarial y el surgimiento de empresarios con nuevas habilidades. Nuevamente, en la década de los años 1990, la globalización de las empresas y de las economías regionales pone en riesgo el crecimiento y el desarrollo de las grandes potencias, $y$ surgen nuevos y aún poco conocidos actores mundiales. Las fronteras de los países se abren, el intercambio de mercancías crece de manera considerable y surgen grandes oportunidades para la puesta en marcha, el crecimiento y desarrollo de nuevas iniciativas empresariales. El mercado ya no es una localidad, región o nación. Gracias al desarrollo de la tecnología, jel mercado es todo el mundo!

\section{El proceso emprendedor}

A fines del siglo XIX, era clara la importancia de las empresas para el desarrollo de las naciones, la importancia de los empresarios para el desarrollo de las empresas y la importancia de ciertos atributos y cualidades personales para el éxito de los empresarios. Sin embargo, también era evidente la necesidad de estudiar, desde una perspectiva empresarial, los factores psicológicos y no psicológicos de los empresarios que influyen en el éxito de las empresas y, en consecuencia, en la generación de valor para la sociedad. A lo largo de los años, quedó claro que el marco conceptual del entrepreneurship implica un estudio de:

- El entorno empresarial que promueva nuevos emprendimientos

- La capacidad del empresario para identificar oportunidades
- Las características del empresario para liderar

- Las herramientas necesarias para la organización de la empresa

- Las características del empresario para gestionar con éxito la organización.

El estudio del empresario, por si solo, no es suficiente para comprender ni dar explicaciones del quehacer de las empresas. Para comprender al empresario y descubrir por qué algunas empresas son exitosas y otras no, algunos estudiosos (Venkataraman 1997, Shane 2003) sugieren que las investigaciones se basen en el estudio del proceso emprendedor y no solo en el análisis de las características de los empresarios.

Según Shane (2003), el entrepreneurship debe ser analizado como un proceso que involucra el estudio de la oportunidad empresarial (mediante un análisis del entorno), los individuos que identifican la oportunidad y toman la decisión de explotarla (los empresarios), las habilidades y estrategias utilizadas para organizar y explotar las oportunidades (los expertos con conocimientos), y las condiciones del ambiente que favorecen la puesta en marcha y el desarrollo de nuevas iniciativas empresariales (el entorno). En este sentido, Shane hace un profundo y comprensible análisis de la relación entre el «individuo» y la «oportunidad», como parte central del «proceso emprendedor». Para Shane, «las oportunidades de negocio existen» independientemente de los individuos que hay en el sistema, razón por la cual hay países con gran riqueza aún poco explotada por los individuos que componen esos países. Shane afirma que son los «individuos», y no los grupos o las empresas, quienes descubren las oportunidades. En este sentido, los «individuos» son parte importante del análisis del proceso emprendedor.

En cuanto al estudio de las oportunidades, las dos perspectivas más comunes son las de Kirzner (1997) y la de Schumpeter (1997). Para el primero, la existencia de una oportunidad depende del conocimiento que los individuos tengan de información ya existente; mientras que para el 
segundo, la nueva información será la que explique la existencia de oportunidades empresariales. Otro aspecto importante de las oportunidades es que estas se generan, fundamentalmente, por cambios tecnológicos, políticos/legales y sociales/demográficos. Estas oportunidades pueden manifestarse creando nuevos productos o servicios, desarrollando nuevas formas de organizarse, descubriendo nuevas materias primas o recursos, identificando nuevos mercados o introduciendo nuevos procesos productivos.

Con respecto a las personas que identifican la oportunidad, toman la decisión de explotarla y se organizan para hacerlo, Shane (2003) señala que existen factores personales, psicológicos y no psicológicos, que influyen en el proceso emprendedor y, por lo tanto, en la posibilidad de descubrir una oportunidad de negocio y llevarla a cabo de manera exitosa. Como factores no psicológicos, considera la educación, la experiencia profesional, la edad, el estado civil, la posición social y el costo de oportunidad. Como factores psicológicos, considera aspectos de personalidad y motivación, el conocimiento de sus propias capacidades y características cognitivas como la confianza en sí mismo, representatividad en su medio e intuición para tomar decisiones en ambientes complejos y dinámicos, donde la información rara vez es completa y suficiente.

El análisis del ambiente y de la industria también forma parte del proceso emprendedor, pues de él dependerá la existencia de oportunidades y la percepción de riesgo por parte del emprendedor. Para Shane y Khuranam (2001), los niveles de conocimiento en una industria en particular, la demanda en un sector empresarial, el ciclo de vida de la industria, las condiciones de la propiedad (patentes, activos, recursos) y la estructura de la industria son factores que motivarán el espíritu emprendedor de los potenciales empresarios y promoverán o no la creación de nuevas empresas.

La financiación es otro de los aspectos fundamentales del proceso emprendedor. Para aprove- char oportunidades se necesitan recursos y estos deben ser adquiridos antes de iniciar las ventas. Los instrumentos financieros para financiar nuevas empresas son múltiples y dependen de las características particulares de cada economía. Sin embargo, la asimetría de información entre el empresario y los potenciales inversionistas es una variable que afecta a todas las nuevas oportunidades de empresa.

Obtenidos los recursos financieros necesarios, el siguiente paso es identificar las estrategias que el emprendedor utilizará para organizarse y crear una ventaja competitiva en el menor tiempo posible. Con el establecimiento de los procesos, las rutinas y las estructuras necesarias para combinar los recursos de manera eficiente, el empresario crea valor para todos los grupos de interés vinculados al negocio.

Finalmente, el proceso emprendedor concluirá con la constitución formal de la empresa, el reclutamiento, la selección y la contratación de colaboradores, el establecimiento de las políticas, los procesos y la estructura organizacional, necesarios para el logro de los objetivos previamente establecidos en función de la visión del emprendedor.

En cada una de estas etapas del proceso emprendedor, el empresario es el actor principal. Sin embargo, no se puede estudiar su comportamiento de manera aislada. Es necesario considerar el entorno, las oportunidades, la organización, el equipo de trabajo y, por supuesto, las características y el estilo personal de cada empresario.

\section{Emprendimientos por necesidad o por oportuni- dad}

Según el Global Entrepreneurship Monitor (2005), conocido como GEM por sus siglas en inglés, los individuos suelen participar en actividades empresariales por dos razones fundamentales. La primera, para explotar una oportunidad de negocio identificada previamente y la segunda, porque no tiene otra opción de trabajo o las opcio- 
nes existentes no satisfacen las necesidades del individuo. En este sentido, según el reporte gerencial del año 2004 , el $65 \%^{3}$ de los individuos involucrados en nuevas aventuras empresariales en ese año, lo hicieron con la finalidad de aprovechar una oportunidad; mientras que el 35\% lo hizo porque no tenía otra opción de empleo. Sin embargo, existe una gran variabilidad entre los 34 países materia del estudio. Mientras que la tasa de actividad empresarial por oportunidad, fue en promedio del 6,2\% en el ámbito mundial; en el Perú, esta tasa fue del $27 \%$ en ese año (GEM 2005). Por otro lado, mientras que la TEA por necesidad fue de $2,3 \%$ en el nivel de la muestra; en el Perú, esta fue equivalente al $13,1 \%$, es decir, aproximadamente uno de cada siete trabajadores, entre 18 y 64 años de edad, era empresario por necesidad en el año 2004.

El mismo consorcio de investigación, en su reporte del año 2007 (Bosma et al. 2008), confirma que el surgimiento de nuevas empresas por oportunidad es mayor en los países desarrollados que en los países en vías de desarrollo. En países con bajos niveles de producción y empleo, y donde la seguridad social es aún incipiente, el desarrollo de nuevos emprendimientos podría ser la única alternativa para generar ingresos y bienestar. GEM también señala que si bien la creación de nuevas empresas por necesidad contribuye con la generación de nuevos puestos de trabajo, las iniciativas empresariales que perduran en el tiempo (GEM considera 3,5 años) son las que mostrarían un mejor desempeño del empresario durante el proceso emprendedor. En este sentido, GEM establece tres etapas en el proceso empresarial. Una primera etapa se inicia con la concepción y puesta en marcha de la empresa, y suele ser menor a tres meses; una segunda etapa se refiere al crecimiento de la nueva empresa y comprende hasta 3,5 años de las empresas. Estas dos primeras etapas son conocidas como Actividad Empresarial en Eta- pa Temprana o «TEA» ${ }^{4}$. GEM reconoce una tercera etapa denominada etapa de persistencia, en la que el propietario-gerente de una empresa ya establecida por más de 3,5años, aún continúa en el mercado, a pesar de que sus productos ya no son necesariamente vistos como una novedad.

Finalmente, reconociendo la importancia del rol del empresario, la necesidad de estudiar el proceso emprendedor y la relevancia de un «empresario por oportunidad» para la generación de riqueza, a continuación, se presenta la metodología empleada para desarrollar la investigación que permitió dar respuesta a la siguiente pregunta: ¿cuáles son los factores que los empresarios entrevistados consideran fundamentales para alcanzar el éxito empresarial?

\section{Metodología}

Con la finalidad de conocer las características fundamentales para alcanzar el éxito empresarial, se realizaron 99 entrevistas en profundidad a empresarios peruanos, residentes en la ciudad de Lima, que tuvieran estudios universitarios y que se considerasen exitosos. A cada uno de ellos, se le realizó una entrevista para obtener:

Datos generales del empresario: género, edad, nivel de formación, entorno familiar, fortalezas y debilidades personales.

Datos generales de la empresa: cuándo y bajo qué circunstancias se formó la empresa, quiénes son los socios fundadores, quienes son los socios actuales, qué productos o servicios ofrece, quiénes son sus actuales y potenciales clientes, cómo ha evolucionado la empresa en los últimos años, cómo se ve la empresa en el futuro, cómo está en términos económicos y de mercado, cuáles son las principales fortalezas y debilidades, cuáles son las mayores oportunidades y amenazas.

3. El estudio es realizado en base a 34 países.

4. La TEA es un índice que consiste, esencialmente, en la suma de nuevos empresarios (aquellos individuos, entre 18 y 64 años de edad, que han tenido alguna acción para crear una empresa en el último año, que han pagado sueldos y salarios por no más de tres meses, y que esperan compartir la propiedad del nuevo negocio) y nuevas empresas (aquellas empresas que han pagado sueldos y salarios por un mínimo de tres meses y un máximo de 42 meses). 
Circunstancias en las que se crea la empresa: características del ambiente externo al inicio de las actividades empresariales, descubrimiento de una oportunidad, necesidad de generar una fuente de ingresos propia, deseo de ser empresario para gozar de independencia y mayor libertad en la toma de decisiones.

Principales inconvenientes a lo largo de su labor empresarial: cuáles fueron los principales, en qué circunstancias se dieron, cómo se superaron.

Visión empresarial: cómo ve el futuro de su empresa, cómo ve su futuro como empresario-; si hoy pudiera escoger entre ser empleado o empresario, cuál de las alternativas escogería y por qué. Valoración del éxito: en qué medida el empresario se considera exitoso, cuál es el factor fundamental para haber logrado el éxito, cuál es su principal cualidad gracias a la cual ha sido capaz de crear este negocio y llevarlo adelante.

Percepción de la empresarialidad en el país: cómo ve el futuro de la clase empresarial peruana, qué tipo de formación cree que necesita un joven peruano para ser un empresario exitoso, qué consejo le daría a los que empiezan a crear su empresa, cree que hay grandes entre los empresarios de las pequeñas, medianas y grandes empresas.

Factores claves para de éxito empresarial: qué capacidad o atributo personal lo llevó al éxito empresarial.

Cabe señalar que cada uno de los elementos de la muestra debería cumplir con los siguientes requisitos:

1. Ser fundador capitalista y gerente de la empresa en la que actualmente está trabajando. De esta manera, el empresario cumplía cabalmente con el rol económico y gestor.

2. Tener el grado académico de bachiller en cualquier especialidad, con lo cual la formación profesional dejaba de ser un diferenciador entre los entrevistados.

3. Haber tenido experiencia profesional como dependiente, no menor a dos años, antes de haber puesto la empresa en marcha. Así, la experiencia dejaba de ser una variable diferenciadora de la muestra.

4. Que la empresa haya tenido un ingreso promedio anual, en el año 2007, mayor a 525,000 nuevos soles ${ }^{5}$.

5. Que la empresa tenga una antigüedad no menor a 3,5años al momento de realizar la entrevista al empresario. De esta manera, la empresa habría superado la etapa conocida como Actividad Empresarial en Etapa Temprana, poniendo a prueba las habilidades y competencias de sus fundadores.

Al cumplir con estos requisitos, los elementos de la muestra tuvieron un alto grado de homogeneidad, lo que permitió aislar la variable factores de éxito empresarial.

\section{Aplicación de la metodología}

Entre los meses de abril y setiembre del presente año, se desarrollaron 99 entrevistas en profundidad a empresarios elegidos por referencias y que cumplieran con los requisitos previamente establecidos. Del total de entrevistados, 20 fueron empresarias mujeres y 79 fueron varones. Del total de mujeres el $50 \%$ tenía entre 40 y 50 años de edad; mientras que del total de varones, el $41 \%$ tenía entre 50 y 60 años de edad (véase el gráfico 1). A lo largo de las entrevistas, fue posible observar que las mujeres profesionales suelen comenzar una aventura empresarial a una edad promedio menor que los varones profesionales. Durante las entrevistas se puedo comprobar que para las mujeres profesionales, mantener un equilibrio entre la actividad profesional y el rol de madre y esposa no era una tarea fácil. Además, para la mayoría de ellas dejar de trabajar no era una alternativa interesante ni viable por razones económicas y de desarrollo personal. En consecuencia, convertirse en empresaria independiente, con mayor libertad para manejar sus tiempos y responsabilidades, fue una opción de trabajo interesante para lograr un equilibrio entre la vida personal y profesional.

5. A partir del año 2008, se considera microempresa a aquella cuyas ventas anuales sean menores a S/. 525.000. 
Gráfico 1

Distribución porcentual de encuestados según edad y sexo

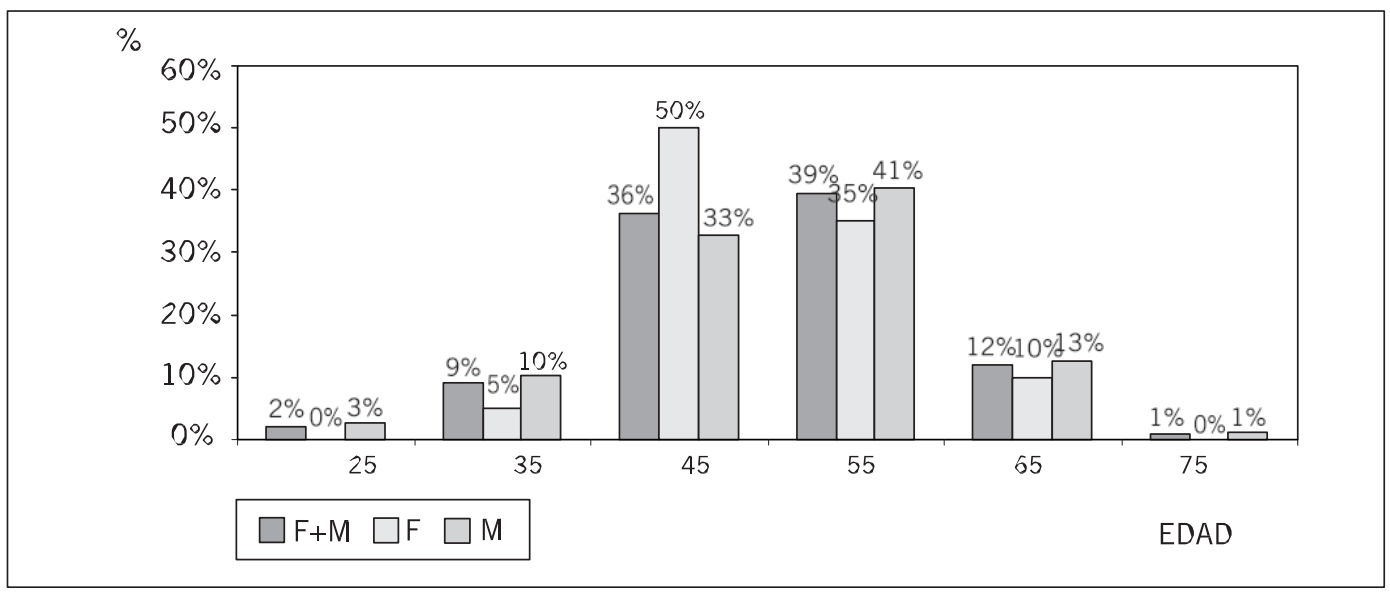

Fuente: elaboración propia.

\section{Motivación para la creación de empresa}

En cuanto a las razones que motivaron a los entrevistados a poner una empresa en marcha, el $44 \%$ mencionó que inició una empresa porque observó una oportunidad de negocio y decidió explotarla (véase el gráfico 2). El $42 \%$ lo hizo porque decidió ser empresario, y buscó una oportunidad que le permitiera obtener los beneficios deseados y un «estilo de vida» propio. Un 13\% de los entrevistados mencionó que tuvo que poner una empresa en marcha por la necesidad de obtener mayores ingresos y manejar sus propios tiempos.

Todos los empresarios tenían la experiencia profesional y los conocimientos necesarios, previo a la puesta en marcha de la empresa. Ninguno de

\section{Gráfico 2}

\section{Motivación para ser empresario/a}

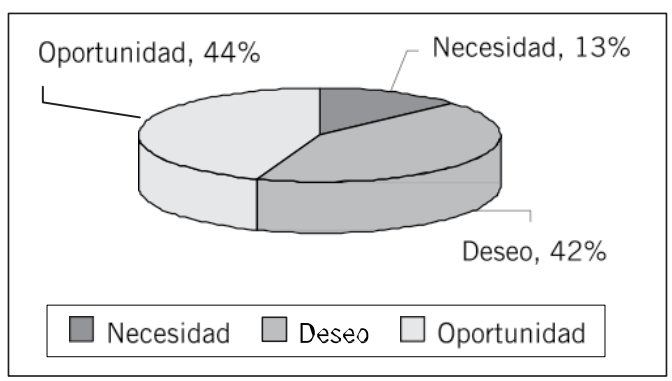

Fuente: elaboración propia. ellos se vio obligado a poner una empresa por razones de sobrevivencia o única opción de generación de ingresos.

En el caso de las mujeres, 10 de ellas señalaron que se convirtieron en empresarias por el «deseo» de serlo, mientras que 7 ingresaron porque «descubrieron una oportunidad» y 3 lo hicieron por «necesidad» (véase el gráfico 3 ).

\section{Gráfico 3}

Motivación para ser empresaria

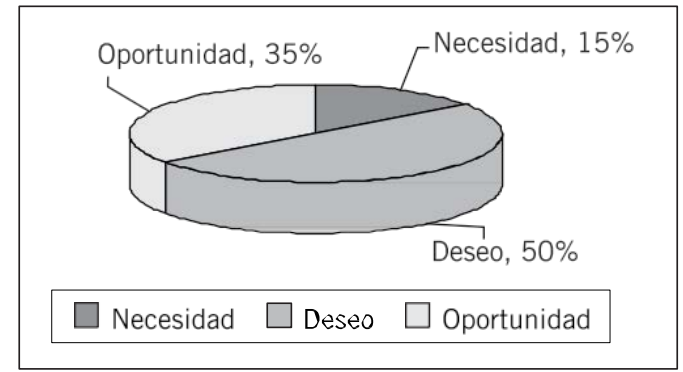

Fuente: elaboración propia.

En el caso de los varones, 37 de los profesionales ingresaron al mundo de los empresarios porque «descubren una oportunidad» $\mathrm{y}$ «deciden explotarla»; 32 lo hacen porque tienen el «deseo» de hacerlo, mientras que 10 lo hacen por «necesidad», ya sea por la necesidad de gene- 
rar mayores ingresos o por el deseo de ser independiente y manejar sus propios tiempos (véase el gráfico 4).

\section{Gráfico 4}

\section{Motivación para ser empresario}

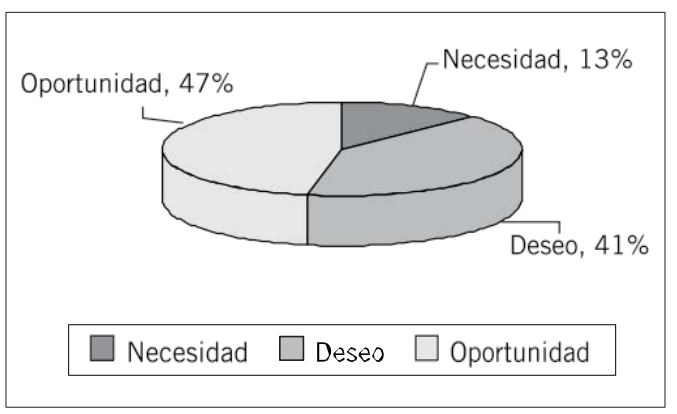

Fuente: elaboración propia.

Durante las entrevistas se pudo observar que los hombres profesionales reconocen tener mayores oportunidades, para iniciar una nueva iniciativa empresarial, que las mujeres profesionales. A los varones se les presentan las oportunidades, mientras que las mujeres tienen que buscarlas con mayor ahínco. Las mujeres buscan oportunidades que les permitan un trabajo independiente, con horarios flexibles y el establecimiento de objetivos en función de las circunstancias persona- les de cada una de ellas. Solo entonces, la experiencia empresarial se convierte en una alternativa interesante para aquella profesional y empresaria que tiene a su cargo la responsabilidad de un hogar.

\section{Actividad empresarial por sector}

En líneas generales, del total de hombres y mujeres entrevistados, más del $50 \%$ se ubica en el sector servicios (véase el cuadro 2). Este era un resultado de esperarse, debido a que la mayoría de las empresas en Lima son de servicios. Sin embargo, se observa que los empresarios varones son quienes se ubican, principalmente, en los sectores agrícola y de construcción; mientras que las empresarias mujeres se vinculan a actividades de servicios sociales y de salud.

También se observó que más del 70\% de los entrevistados formó una empresa porque descubrió una oportunidad de negocio en un sector en el que tenía experiencia laboral. Esto permite suponer que la formación profesional y la experiencia laboral posterior a los estudios universitarios, serían determinantes en la elección del sector o industria en la que el empresario decida poner su empresa.

\section{Cuadro 2}

Actividad empresarial por sector

\begin{tabular}{|c|c|c|c|c|c|}
\hline \multirow{2}{*}{ Sector } & \multirow{2}{*}{$\begin{array}{l}\text { Total } \\
\text { entrevistados }\end{array}$} & \multicolumn{2}{|c|}{ Total } & \multicolumn{2}{|c|}{ Porcentaje } \\
\hline & & $\boldsymbol{F}$ & $M$ & $F$ & $M$ \\
\hline Agricultura ganadería, caza y silvicultura & 4 & 0 & 4 & $0 \%$ & $5 \%$ \\
\hline Industrias manufactureras & 14 & 2 & 12 & $10 \%$ & $15 \%$ \\
\hline Construcción & 8 & 1 & 7 & $5 \%$ & $9 \%$ \\
\hline $\begin{array}{l}\text { Comercio al por mayor y al por menor, reparación de vehículos } \\
\text { automotores, motocicletas }\end{array}$ & 20 & 3 & 17 & $15 \%$ & $22 \%$ \\
\hline Hoteles y restaurantes & 5 & 2 & 3 & $10 \%$ & $4 \%$ \\
\hline Transporte, almacenamiento y comunicaciones & 5 & 2 & 3 & $10 \%$ & $4 \%$ \\
\hline Actividades inmobiliarias, empresariales y de alquiler & 28 & 4 & 24 & $20 \%$ & $30 \%$ \\
\hline Enseñanza (privada) & 4 & 2 & 2 & $10 \%$ & $3 \%$ \\
\hline Actividades de servicios sociales y de salud (privada) & 6 & 3 & 3 & $15 \%$ & $4 \%$ \\
\hline \multirow[t]{2}{*}{ Otras actividades de servicios comunitarias, sociales y personales } & 5 & 1 & 4 & $5 \%$ & $5 \%$ \\
\hline & 99 & 20 & 79 & $100 \%$ & $100 \%$ \\
\hline
\end{tabular}

Fuente: elaboración propia. 


\section{Percepción del futuro de la empresa}

La percepción del futuro de la empresa está muy vinculada con la visión del empresario. En cuanto a la percepción que la empresaria tenía sobre el futuro de su empresa, todas, salvo una, lo consideraban positivo (véase el gráfico 5). Esta misma empresaria no se consideraba exitosa.

\section{Gráfico 5}

Distribución porcentual de mujeres entrevistadas: ¿cómo considera el futuro de su empresa?

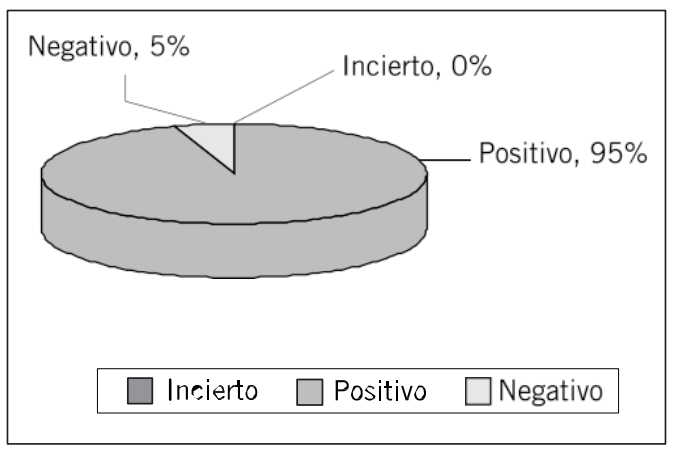

Fuente: elaboración propia.

Entre los varones, la percepción era positiva para el $97 \%$ de los casos e incierta para el $3 \%$ de los mismos.

\section{Gráfico 6}

Distribución porcentual de varones entrevistados: ¿cómo considera el futuro de su empresa?

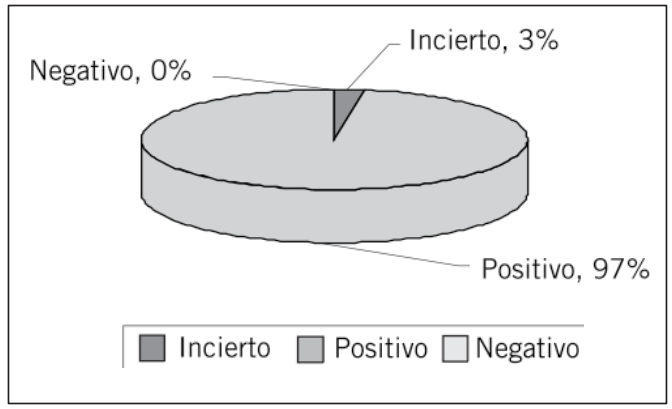

Fuente: elaboración propia.

En líneas generales, la percepción sobre el futuro de la empresa fue muy positiva, porque consi- deraban que el Perú estaba atravesando por una etapa de crecimiento y desarrollo económico muy favorable. Todos los entrevistados mostraron optimismo, incluidos aquellos que veían negativo e incierto el futuro de sus actuales empresas.

\section{Gráfico 7}

Distribución porcentual del total de entrevistados: ¿cómo considera el futuro de su empresa?

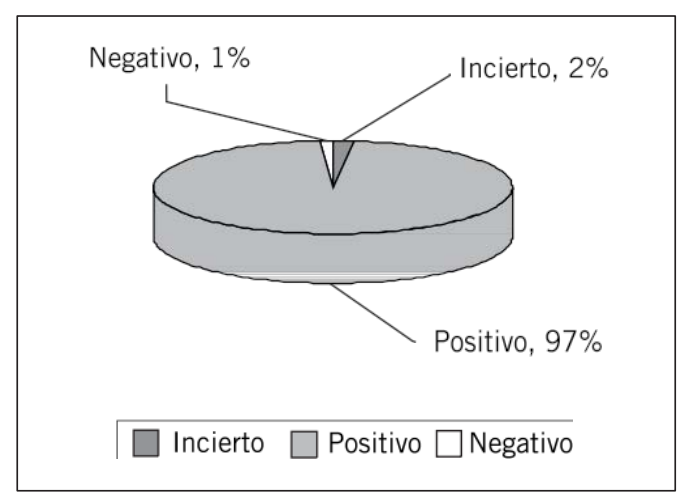

Fuente: elaboración propia.

\section{Factores personales de éxito}

Para conocer cuáles fueron los factores personales que contribuyeron con el éxito empresarial alcanzado, al entrevistado se le hizo una pregunta abierta, para que pueda responder lo que se le viniera a la mente. En total se tuvieron 74 atributos diferentes y, en la mayoría de los casos, el empresario dio tres respuestas.

Tanto varones como mujeres consideran que la perseverancia es la característica fundamental del empresario exitoso. La perseverancia fue la característica más nombrada (34 respuestas), seguida del esfuerzo y la dedicación (8 respuestas). En tercer lugar de preferencia quedó la actitud trabajadora (2), para el caso de las empresarias y la formación académica y profesional (6), para el caso de los empresarios. En consecuencia, la perseverancia, el esfuerzo y la dedicación, una actitud trabajadora y la formación académica y profesional, son los factores de éxito considerados más importantes por los entrevistados (véase el cuadro 3). 
Cuadro 3

Primer factor de éxito

\begin{tabular}{|c|c|c|}
\hline Total & Femenino & Masculino \\
\hline Perseverancia & Perseverancia & Esfuerzo / Dedicacion \\
\hline Esfuerzo / Dedicacion & Esfuerzo / Dedicacion & Formacion Academica/ Profesional \\
\hline Formacion Academica / Profesional & Actitud Trabajadora & Fona \\
\hline
\end{tabular}

Fuente: elaboración propia.

Cuando se pidió a los entrevistados que dieran un segundo factor de éxito, del total de personas, 8 mencionaron la perseverancia como segundo factor de éxito, 5 la actitud trabajadora y 5 la paciencia. Finalmente, frente a un tercer factor de éxito, 9 personas mencionaron la perseverancia como tercer factor de importancia, 5 personas mencionaron la honestidad y 4, el esfuerzo y dedicación.

Los resultados muestran que la perseverancia es la característica fundamental del empresario peruano que ha alcanzando el éxito.

En cuanto a la percepción de «éxito», todos los empresarios coincidieron en señalar que, si bien sentían que habían alcanzado los objetivos planteados, aún les quedaba mucho por hacer. Reconocieron la importancia de la formación profesional adquirida y la experiencia profesional previa a la puesta en marcha de la empresa, como esenciales para haber alcanzado los resultados esperados.

Al realizar el cruce de rangos de edades y factores de éxito, se encontró que del total de entrevistados, hombres y mujeres, la perseverancia predomina como el factor de éxito con más frecuencia en los diferentes rangos de edades que van desde los 20 a menos de 80 años de edad.
Los picos más altos se presentan entre los empresarios cuyas edades se encuentran entre los 40 y 60 años (véase el cuadro 4).

Si se analiza el cuadro 4 con mayor profundidad y detalle, se puede observar que los jóvenes entrevistados menores de 30 años perciben la perseverancia, la paciencia, la responsabilidad y la toma de riesgos como factores de éxito. Según los jóvenes entrevistados, ellos son vehementes y apresurados, por lo cual la paciencia es necesaria; aún están en proceso de crecimiento y maduración, por lo que requieren importantes dosis de responsabilidad; y por ser jóvenes, aún no han acumulado grandes riquezas ni mayor valor intangible. Por lo tanto, como tienen poco que perder, se arriesgan.

Dentro del grupo de empresarios cuyas edades van entre los 40 y 60 años, se encuentra a la perseverancia como la variable con más frecuencia en los tres factores de éxito principales, además de la paciencia, el tener una actitud trabajadora, asumir el liderazgo, innovar constantemente, buscar oportunidades y ser honesto.

Al analizar la carrera profesional del empresario, el sector económico en el que se desempeña y el atributo que consideraba fundamental para alcanzar el éxito empresarial (véase el cuadro 5) se llegaron a los siguientes resultados. 


\section{Cuadro 4}

\section{Distribución de factores de éxito por edades}

\begin{tabular}{|c|c|c|c|c|c|}
\hline & \multirow{2}{*}{\multicolumn{3}{|c|}{ Femenino y masculino }} \\
\hline & & & & & \\
\hline Edad & Cant. & Porcent. & $F 1$ & $F 2$ & F3 \\
\hline $\begin{array}{l}\text { De } 20 \text { a } \\
\text { menos de } 30\end{array}$ & 2 & $2 \%$ & (02) perseverancia & $\begin{array}{l}\text { (01) paciencia, } \\
\text { responsabilidad }\end{array}$ & (01) tomar riesgos \\
\hline $\begin{array}{l}\text { De } 30 \text { a } \\
\text { menos de } 40\end{array}$ & 9 & $9 \%$ & $\begin{array}{l}\text { (01) optimismo, actitud } \\
\text { trabajadora, perseveran- } \\
\text { cia, gusto por lo que hace, } \\
\text { busqueda de oportunida- } \\
\text { des, orientacion a identi- } \\
\text { ficar y satisfacer las nece- } \\
\text { sidades de los clientes, } \\
\text { esfuerzo / dedicacion, co- } \\
\text { nocimiento del negocio, } \\
\text { apoyo moral / emocional } \\
\text { de terceros }\end{array}$ & $\begin{array}{l}\text { (01) optimismo, orden y } \\
\text { organización, perseve- } \\
\text { rancia, deter-minacion, } \\
\text { esfuerzo / dedicacion, } \\
\text { creatividad, formacion } \\
\text { acade-mica / profesional, } \\
\text { proactividad, sacrificio }\end{array}$ & $\begin{array}{l}\text { (01) orden y organiza- } \\
\text { ción, capital humano, } \\
\text { orientacion a identifi- } \\
\text { car y satisfacer las ne- } \\
\text { cesidades de los } \\
\text { clientes, factores divi- } \\
\text { nos, red de contactos, } \\
\text { actitud de aprendiza- } \\
\text { je constante, pensar } \\
\text { estrategicamente. }\end{array}$ \\
\hline $\begin{array}{l}\text { De } 40 \text { a } \\
\text { menos de } 50\end{array}$ & 36 & $36 \%$ & (14) perseverancia & $\begin{array}{l}\text { (04) paciencia, perseve- } \\
\text { rancia }\end{array}$ & (05) perseverancia \\
\hline $\begin{array}{l}\text { De } 50 \text { a } \\
\text { menos de } 60\end{array}$ & 39 & $39 \%$ & (13) perseverancia & $\begin{array}{l}\text { (03) actitud trabaja- } \\
\text { dora,liderazgo, innova- } \\
\text { cion constante }\end{array}$ & $\begin{array}{l}\text { (03) perseverancia, } \\
\text { busqueda de oportuni- } \\
\text { dades, honestidad }\end{array}$ \\
\hline $\begin{array}{l}\text { De } 60 \text { a } \\
\text { menos de } 70\end{array}$ & 12 & $12 \%$ & (04) perseverancia & $\begin{array}{l}\text { (02) orientacion a iden- } \\
\text { tificar y satisfacer las } \\
\text { necesidades de los clien- } \\
\text { tes, responsabilidad }\end{array}$ & $\begin{array}{l}\text { (01) optimismo, perse- } \\
\text { verancia, capital huma- } \\
\text { no, orientacion a iden- } \\
\text { tificar y satisfacer las } \\
\text { necesidades de los } \\
\text { clientes, esfuerzo } \\
\text { dedicacion, trabajo en } \\
\text { equipo, responsabili- } \\
\text { dad, honestidad, acti- } \\
\text { tud profesional, humil- } \\
\text { dad }\end{array}$ \\
\hline $\begin{array}{l}\text { De } 70 \text { a } \\
\text { menos de } 80\end{array}$ & 1 & $1 \%$ & $\begin{array}{l}\text { (01) capacidades y habi- } \\
\text { lidades personales }\end{array}$ & $\begin{array}{l}\text { (01) atreverse a empren- } \\
\text { der una empresa }\end{array}$ & $\begin{array}{l}\text { (01) confianza en si } \\
\text { mismo }\end{array}$ \\
\hline TOTAL & 99 & $100 \%$ & $(\#)$ : Frecuencia por cada fa & actor de éxito & \\
\hline
\end{tabular}

Fuente: elaboración propia. 


\section{Cuadro 5}

Relación de los sectores a los que pertenecen

\begin{tabular}{|l|l|}
\hline COD & \multicolumn{1}{c|}{ SECTOR } \\
\hline A & Agricultura ganadería, caza y silvicultura \\
D & Industrias manufactureras \\
F & Construcción \\
G & Comercio al por mayor y al por menor, reparación de vehículos automotores, motocicletas \\
H & Hoteles y restaurantes \\
I & Transporte, almacenamiento y comunicaciones \\
K & Actividades inmobiliarias, empresariales y de alquiler \\
M & Enseñanza(privada) \\
N & Actividades de servicios sociales y de salud (privada) \\
O & Otras actividades de servicios comunitarias, sociales y personales \\
\hline
\end{tabular}

Fuente: elaboración propia.

El $60 \%$ de los empresarios entrevistados son: administradores, economistas, contadores o ingenieros (véase el cuadro 6). La mayoría de los administradores, economistas o contadores trabaja en el sector de "actividades inmobiliarias, empresariales y de alquiler"; mientras que la mayoría de los ingenieros se desempeña en el sector de "comercio al por mayor y menor, reparación de vehículos automotores y motocicletas" (véase el cuadro 7).

\section{Cuadro 6 \\ Clasificación de las carreras profesionales a las que pertenecen los entrevistados}

TIPO DE CARRERA / PROFESIÓN:

T1: administración, economía, contabilidad

T2: ingenierías

T3: derecho, educación, sicología, antropología T4: medicina, biología, odontología, veterinaria Otros

Fuente: elaboración propia.

\section{Cuadro7}

Tabla de distribución de entrevistados según tipo de carrera y sector económico

\begin{tabular}{|l|c|c|c|c|c|c|c|c|c|c|c|c|}
\hline $\begin{array}{c}\text { Carrera I } \\
\text { profesion (tipo) }\end{array}$ & Cant. & Porc. & $\boldsymbol{A}$ & $\boldsymbol{D}$ & $\boldsymbol{F}$ & $\boldsymbol{G}$ & $\boldsymbol{H}$ & $\boldsymbol{I}$ & $\boldsymbol{K}$ & $\boldsymbol{M}$ & $\boldsymbol{N}$ & $\boldsymbol{O}$ \\
\hline T1 & 34 & $34 \%$ & 2 & 6 & 2 & 6 & 2 & 3 & 11 & 0 & 0 & 2 \\
T2 & 26 & $26 \%$ & 1 & 5 & 4 & 8 & 1 & 0 & 5 & 0 & 0 & 2 \\
T3 & 9 & $9 \%$ & 0 & 1 & 0 & 1 & 0 & 0 & 4 & 3 & 0 & 0 \\
T4 & 8 & $8 \%$ & 1 & 0 & 0 & 2 & 0 & 1 & 0 & 0 & 4 & 0 \\
OTROS & 22 & $22 \%$ & 0 & 2 & 2 & 3 & 2 & 1 & 8 & 1 & 2 & 1 \\
\hline TOTAL & 99 & $100 \%$ & 4 & 14 & 8 & 20 & 5 & 5 & 28 & 4 & 6 & 5 \\
\hline
\end{tabular}

Fuente: elaboración propia. 
Para la mayoría de ellos, la perseverancia es un atributo personal fundamental para alcanzar el éxito. Sin embargo, es interesante observar en el cuadro 8 el detalle de las respuestas que dieron los entrevistados de estas carreras mayoritarias.

La revisión bibliográfica hace referencia a la importancia que tiene el entorno para la creación de nuevas iniciativas empresariales. Por lo general, en momentos en el que Estado promueve la creación de nuevas empresas facilitando trámites burocráticos, brindado acceso a líneas de crédito y promoviendo la innovación y la tecnología, los profesionales que ocupan importantes cargos directivos suelen comenzar su vida empresarial por el «deseo» de mejorar sus ingresos, tener mayor grado de libertad y aprovechar la oportunidad de un entorno estable y promotor de nuevas empresas. En este sentido, los ejecutivos deci- den renunciar a sus empleos para buscar y aprovechar alguna oportunidad de mercado.

En el cuadro 9, en el que se cruzan las variables: «antigüedad de la empresa» y «motivo para iniciar una actividad empresarial», es posible observar que el $19 \%$ de las empresas que tienen entre 3,5 y 10 años de antigüedad, fueron creadas por el «deseo del empresario»; mientras que, en el mismo período, el $16 \%$ de las empresas fueron creadas porque el empresario descubrió una oportunidad. Asimismo, los empresarios entrevistados, con empresas que tienen entre $11 \mathrm{y}$ 20 años de antigüedad, se convirtieron en empresarios por deseo (14\%) y por oportunidad (11\%). Finalmente, las empresas que tenían entre 21 y 30 años de existencia, resaltaban la oportunidad (12\%) como motivo principal para la creación de la empresa.

\section{Cuadro 8}

Factores claves de éxito según administradores, economistas, contadores e ingenieros

\begin{tabular}{|c|c|c|c|}
\hline $\begin{array}{l}\text { Carrera / Profesion } \\
\text { (Tipo) }\end{array}$ & $\begin{array}{c}\text { CIIU } \\
\text { (Sector) }\end{array}$ & Cant & F1 \\
\hline T1 & A & 2 & (01) actitud trabajadora, experiencias como empresario o empleado \\
\hline T1 & $\mathrm{D}$ & 6 & (02) perseverancia \\
\hline T1 & $\mathrm{F}$ & 2 & (01) actitud trabajadora, esfuerzo / dedicación \\
\hline T1 & G & 6 & $\begin{array}{l}\text { (01) orden y organización, perseverancia, esfuerzo / dedicación, apoyo } \\
\text { moral / emocional de terceros, formación académica / profesional, no se } \\
\text { amilana ni se rinde con facilidad }\end{array}$ \\
\hline $\mathrm{T} 1$ & $\mathrm{H}$ & 2 & (01) perseverancia, capital humano \\
\hline T1 & I & 3 & (02) perseverancia \\
\hline T1 & $\mathrm{K}$ & 11 & (02) perseverancia, gusto por lo que hace \\
\hline T1 & M & 0 & ----- \\
\hline T1 & $\mathrm{N}$ & 0 & ---- \\
\hline T1 & 0 & 2 & (01) perseverancia, búsqueda de oportunidades \\
\hline $\mathrm{T} 2$ & A & 1 & (01) actitud trabajadora \\
\hline $\mathrm{T} 2$ & $\mathrm{D}$ & 5 & (03) perseverancia \\
\hline $\mathrm{T} 2$ & $\mathrm{~F}$ & 4 & $\begin{array}{l}\text { (01) perseverancia, búsqueda de oportunidades, formación académica / } \\
\text { profesional, capacidad de decisión }\end{array}$ \\
\hline T2 & G & 8 & (04) perseverancia \\
\hline $\mathrm{T} 2$ & $\mathrm{H}$ & 1 & (01) esfuerzo / dedicación \\
\hline $\mathrm{T} 2$ & 1 & 0 & ---- \\
\hline $\mathrm{T} 2$ & $\mathrm{~K}$ & 5 & $\begin{array}{l}\text { (01) gusto por lo que hace, conocimiento del negocio, liderazgo, formacion } \\
\text { académica / profesional, elaboración de un plan de negocios }\end{array}$ \\
\hline T2 & M & 0 & ---- \\
\hline T2 & $\mathrm{N}$ & 0 & ---- \\
\hline $\mathrm{T} 2$ & $\mathrm{O}$ & 2 & (01) oportunismo, honestidad \\
\hline \multicolumn{2}{|l|}{ TOTAL } & 60 & (\#) : Frecuencia por cada factor de éxito \\
\hline
\end{tabular}




\section{Cuadro 9}

Tabla de distribución de entrevistados según motivo por el que se convierte en empresario y antigüedad de la empresa

\begin{tabular}{|lcc|cccccc|}
\cline { 3 - 9 } \multicolumn{1}{c}{} & \multicolumn{6}{c|}{ Antiguedad de la empresa (años) } \\
\hline ¿Empresario por? & Cant & Porc. & $\mathbf{0 - 1 0}$ & $\mathbf{1 1 - 2 0}$ & $\mathbf{2 1 - 3 0}$ & $\mathbf{3 1 - \mathbf { 4 0 }}$ & $\mathbf{4 1}-\mathbf{5 0}$ & $\mathbf{5 1}-\mathbf{6 0}$ \\
\hline Deseo & 42 & $42 \%$ & 19 & 14 & 6 & 3 & 0 & 0 \\
Necesidad & 13 & $13 \%$ & 5 & 5 & 1 & 1 & 0 & 1 \\
Oportunidad & 44 & $44 \%$ & 16 & 11 & 12 & 2 & 3 & 0 \\
\hline Total & $\mathbf{9 9}$ & $\mathbf{1 0 0 \%}$ & $\mathbf{4 0}$ & $\mathbf{3 0}$ & $\mathbf{1 9}$ & $\mathbf{6}$ & $\mathbf{3}$ & $\mathbf{1}$ \\
\hline
\end{tabular}

Fuente: elaboración propia.

Por lo tanto, de las entrevistas realizadas se puede inferir que, a lo largo de los años y dadas las condiciones del entorno, los motivos para emprender una empresa cambian, lo cual se refleja en el nivel de estabilidad y los factores de riesgo vividos en el Perú desde hace treinta años. Es así que la variable oportunidad siempre tendrá un comportamiento casi homogéneo, porque independientemente de lo bien o mal que se encuentre la economía nacional, siempre existirán oportunidades y los empresarios se mantienen alerta a la existencia de cada una de ellas. Sin embargo, el deseo de volverse empresario, generalmente, se presenta en entornos estables con economías en crecimiento.

\section{Conclusiones}

Una investigación cualitativa de tipo exploratoria muestra que la característica fundamental para alcanzar el éxito en el Perú, según empresarios limeños de la pequeña y mediana empresa, es la perseverancia. Sin embargo, el 98\% de los empresarios señaló que la formación académica y la experiencia empresarial previa en alguna empresa del sector fue determinante para identificar una oportunidad de negocio, tomar la decisión de explotarla, organizarse para poner en marcha una iniciativa empresarial y tener resultados positivos. En consecuencia, la formación y la experiencia son indispensables pero no suficientes; la perseverancia debe cultivarse.

En los últimos veinte años, los programas de formación de administradores de empresas han in- corporado una serie de asignaturas orientadas al desarrollo del espíritu emprendedor y del proceso emprendedor, en respuesta a la necesidad de crear empresas innovadoras y nuevos puestos de trabajo, contribuyendo de esta manera con el crecimiento y desarrollo de las naciones. Conocer la percepción de los empresarios, sobre los factores de éxito empresarial en el Perú, es de suma importancia para quienes están en el sector educativo, especialmente en la formación de futuros profesionales y empresarios.

Hoy, las investigaciones nos muestran que en ambientes sumamente cambiantes y competitivos, los empresarios requieren estar académicamente bien preparados y deben gozar de una experiencia laboral enriquecedora antes de iniciar una iniciativa empresarial. Además de los conocimientos y habilidades, el empresario peruano de manera particular- requiere grandes dosis de perseverancia, compromiso y determinación. Mientras que en las economías desarrolladas, la innovación suele ser la característica fundamental del empresario exitoso; en el Perú, la perseverancia, el esfuerzo y la dedicación, la formación académica profesional, la actitud trabajadora, la honestidad y la tolerancia al riesgo, son las actitudes que el empresario debe practicar para alcanzar el éxito empresarial. En más de una oportunidad se «cerrará la puerta» al empresario, pero su perseverancia, esfuerzo, dedicación y honestidad, además de una sólida formación profesional y experiencia de trabajo, contribuirán con el éxito empresarial, aunque no hay nada, ni nadie, que pueda garantizarlo. 


\section{Referencias}

ACS, Z.; P. ARENIUS, M. HAY y M. MINNITI

2005 Global Entrepreneurship Monitor 2004 Global Report. Estados Unidos, Reino Unido: Babson College, London Business School.

BARON, R.

1998 «Cognitive Mechanisms in Entrepreneurship: Why and When Entrepreneurs Think Differently than Other People». En: Journal of Business Venturing, Vol. 13, pp. 275 - 294.

BLANCHFLOWER, D. y A. OSWALD

1998 «What makes an entrepreneur?». En: Journal of Labor Economics, Vol. 16, № 1, pp. 2660.

BOSMA, N.; K. JONES, E. AUTIO y J. LEVIE

2008 Global Entrepreneurship Monitor 2007 Global Report. Estados Unidos, Reino Unido: Babson College, London Business School.

BUYANT, Christian y Pierre-André JULIEN

2000 "Defining the Field of Research in Entrepreneurship». En: Journal of Business Venturing, Vol. 16, pp. $165-180$.

BUSENITZ, Lowell W.; WEST, G.Page; SHEPHERD, Dean; NELSON, Teresa; CHANDLER, Gaylen N.; ZACHARAKIS, Andrew.

2003 «Entrepreneurship Research in Emergence: Past Trends and Future Directions». En: Journal of Management, Vol. 29, $\mathrm{N}^{\circ} 3$, pp. 285-308.

DETIENNE, Dawn R. y Dean A. SHEPHARD

2005 «Prior Knowledge, Potential Financial Reward, and Opportunity Identification». Entrepreneurship: Theory \& Practice Vol. 29, $\mathrm{N}^{\circ} 1, \mathrm{p} 91-112,22 \mathrm{p}$.

GARTNER, W.

1990 «What are we talking about when we talk about entrepreneurship?». En: Journal of Business Venturing, Vol. 5, № 1, pp. 15-29.
GARTNER, W.

1998 «¿Who Is An Entrepreneur? Is the Wrong Question». En: American Journal of Small Business, Vol. 12, pp. 11-32.

GLOBAL ENTREPRENEURSHIP MONITOR (GEM)

2005 Executive Report 2004. Estados Unidos, Reino Unido: Babson College, London Business School.

HEBERT, Robert F. y Albert N. LINK

1998 «In Search of the Meaning of Entrepreneurship», En: Small Business Economics, Vol. 1, pp. 39-49.

1988 The Entrepreneur: Mainstream Views and Radical Critiques. Nueva York: Praeger.

KANTIS, Hugo; ISHIDA, Masahiko y KOMORI, Masahiko

2002 Empresarialidad en economías emergentes: creación y desarrollo de nuevas empresas en América Latina y el Este de Asia. Washington, D.C.: Banco Interamericano de Desarrollo.

KIRZNER, I.M

1997 «Entrepreneurial discovery and the competitive market process: an Austrian approach». Journal of Economic Literature, XXXV (1):60-85

MINNITI M.; W.B. BYGRAVE, E. AUTIO

2006 Global Entrepreneurship Monitor. Executive Report 2005. Estados Unidos, Reino Unido: Babson College, London Business School.

PRAAG, C. y H. POHEM

1995 «Determinants of willingness and opportunity to start as an entrepreneur». En: Kyklos, Vol. 48, No 4 , pp. 513-540.

SCHUMPETER, J. A.

1997 Teoría del Desenvolvimiento Económico. México, Fondo de Cultura Económica. Segunda edición en español 
SHANE, Scott

2003 A General Theory of Entrepreneurship: The Individual-Opportunity Nexus (New Horizons in Entrepreneurship Series). USA: Edward Elgar Publishing.

2000 «Prior Knowledge and the Discovery of Entrepreneurial Opportunities». En: Organiza-tion Science, Vol. 11, No 4, pp. 448-469.

SHANE, S. y R. KHURANAM

2001 «Career experiences and firm founding», documento presentado a la Academy of Management Meetings.

SHANE, S. y S. VENKATARAMAN

2000 «The Promise of Entrepreneurship as a Field of Research». En: Academy of Management Review, Vol. 25, № 1, pp. 217-226.

VAN PRAAG, C. M.

1999 «Some Classic Views on Entrepreneurship». En: The Economist, Vol. 147, № 3, p. 311.
VARELA, Rodrigo

2008 Innovación empresarial: arte y ciencia en la creación de empresas. 3ra. Edición. Colombia: Pearson Educación.

\section{VENKATARAMAN, S.}

1997 «The distinctive domain of entrepreneurship research: An editor's perspective». In: J. Katz and R. Brockhaus (eds), Advances in Entrepreneurship, Firm Emergence, and Growth, 3 Greewinch, CT, US: JAI Press, pp. 119-138.

\section{VILLARÁN, Fernando}

2001 «Competencias necesarias para la creación y gestión exitosa de micro y pequeñas empresas en el Perú». Documento de Trabajo № 1. Lima, Perú: Ministerio de Educación de Perú, Programa Especial Mejoramiento de la Calidad de la Educación Peruana. 